\title{
Estudo comparativo entre três técnicas abertas de orquiectomia em gatos
}

\author{
A comparative study among three open orchiectomy techniques in cats \\ Karen Maciel de Oliveira', Leonardo Augusto Lopes Muzzi ${ }^{2}$, Bruno Benetti Junta Torres' , Endrigo \\ Gabellini Leonel Alves', Gabriela Rodrigues Sampaio² \& Ruthnea Aparecida Lázaro Muzzi²
}

\begin{abstract}
Background: Surgical sterilization of male cats is one of the most commonly procedures in the small animals practice, used as a control method against the overpopulation of domestic cats. Orchiectomy also aims to prevent or treat hormone-mediated diseases, disorders of the reproductive organs, such as testicular cancer, orchitis, prostatic diseases, trauma or abscesses. In addition, it may be used to control undesirable changes in behavior in adult cats such as the case of territorial marking and aggression. Several surgical techniques are described for orchiectomy in cats using different methods of hemostasis and/or ligation of the spermatic cord. There is a paucity of scientific studies and comparative data that supports one technique over the other, so this experiment was conducted to compare the three major open surgical techniques of ligation of the spermatic vessels to perform orchiectomy in cats.

Materials, Methods \& Results: Thirty-nine young entire adult male cats, with an average weight of $2.7 \mathrm{~kg}$, were divided into three groups. In group I, animals underwent orchiectomy using the suture technique to ligation of the spermatic cord. In group II, the spermatic cord was used to perform an "eight-knot" on itself. In group III, the vas deferens and spermatic cord were used together to make "square-knots". The outcome of surgical procedures was assessed by clinical examination, incidence of complications trans and postoperatively, duration of the procedure and ease of implementation. None of the animals had immediate or delayed bleeding after hemostasis. During surgery, there was no significant difference in procedure duration among the three different techniques. Postoperatively, the overall clinical evaluation was normal and there were no changes in behavior in any animal. It was noted a statistically significant difference in respiratory rate among groups, with the GI animals showing high frequency at the postoperative period when compared to animals from GII and GIII. There were no major complications in any of the techniques used and the parameters showed no significant difference among the groups.

Discussion: The three most widely used techniques to perform orchiectomy in cats were evaluated in this study. Even though the respiratory rate has been shown to be statistically different among the groups, it was not a significant factor, because all other parameters were normal. These clinical data reflect the absence of major complications of surgery such as infection, pain, hypotension, hypovolemia or shock. In the current study, only adult cats were used and the structures of the spermatic cord showed strong and efficient ligation of testicular vessels with the techniques used. It was clinically observed that there was more edema in the group whose ligature employed was the suture technique. The catgut acts as a foreign body and interferes with the inflammatory reaction, the time of absorption and wound healing. The "eight-knot" technique is safe and effective, however, requires greater skill of the surgeon when compared to other techniques evaluated. The technique of "square-knot" resulted in less tissue manipulation without damaging the spermatic vessels, demonstrated by the absence of bleeding and minimal inflammatory reaction. This technique was effective, without complications postoperatively and easy to perform. These data suggest that the three techniques are considered easy and efficacious to perform orchiectomy in cats, but the technique of "square-knot" performed seems to be easier.
\end{abstract}

Keywords: orchiectomy, open techniques, castration, cats.

Descritores: orquiectomia, técnica aberta, castração, gatos. 


\section{INTRODUÇÃO}

A esterilização cirúrgica de gatos machos é um dos procedimentos mais comumente realizados na prática veterinária de pequenos animais, sendo um método eletivo no controle populacional. Também previne patologias hormônio-mediadas, neoplasias testiculares e uma série de mudanças comportamentais indesejáveis no gato adulto, como marcação territorial, hábitos noturnos e agressividade [14]. Além de preventiva, a orquiectomia é utilizada para o tratamento de patologias reprodutivas, como neoplasias testiculares e escrotais, orquites, doenças prostáticas, trauma ou abscessos e controle de alterações endócrinas $[3,6,10,14,15,23]$.

Por ser uma técnica operatória relativamente simples, é realizada com frequência por práticos ou profissionais que não empregam os princípios fundamentais da técnica cirúrgica asséptica [5]. A falta de atenção à higiene e à hemostasia adequada dos vasos pode resultar em complicações pós-operatórias importantes, como infecção, hemorragia e até óbito do animal.

São descritas técnicas cirúrgicas para orquiectomia em gatos nas quais distintos métodos para ligadura e hemostasia do cordão espermático são utilizados. A oclusão dos componentes do cordão espermático pode ser realizada por meio de ligadura com fio cirúrgico [1], utilização de nó em figura de oito empregando-se o próprio cordão espermático $[2,8,13]$ ou utilização de nós quadrados [17]. Pela escassez de dados científicos que permitam apoiar uma técnica em detrimento de outra [25], objetivou-se com esse estudo comparar as três principais técnicas cirúrgicas abertas de ligadura dos vasos espermáticos para realização de orquiectomia em gatos adultos.

\section{MATERIAIS E MÉTODOS}

Este estudo foi aprovado pela Comissão de Avaliação de Projetos da Universidade Federal de Lavras (UFLA), protocolo $n^{\circ} 48 / 2006$. Foram utilizados 39 gatos machos inteiros, adultos jovens, sem raça definida, com peso corpóreo médio de $2,7 \mathrm{~kg}$ $( \pm 1,5)$, clinicamente saudáveis, apresentados ao serviço de cirurgia da Universidade Federal de Lavras para orquiectomia eletiva. Os felinos foram avaliados e selecionados por meio de exame clínico préoperatório e hemograma completo.
No período pré-cirúrgico, os animais foram submetidos a jejum alimentar de 12 horas e hídrico de quatro horas. Os gatos receberam antibioticoprofilaxia profilática, 30 minutos antes da cirurgia, à base de penicilina benzatina ${ }^{1}(30.000 \mathrm{UI} / \mathrm{kg}, \mathrm{IM})$. Foi utilizada como medicação pré-anestésica a acepromazina $^{2}(0,1 \mathrm{mg} / \mathrm{kg}, \mathrm{IM})$ e para indução e manutenção, anestesia dissociativa com cetamina ${ }^{3}$ $(12 \mathrm{mg} / \mathrm{kg})$ associado a xilazina ${ }^{4}(1 \mathrm{mg} / \mathrm{kg})$ por via intramuscular.

Após tricotomia ampla da região inguinoescrotal, os animais foram posicionados em decúbito dorsal e preparados para cirurgia asséptica. A antissepsia foi realizada com iodopovidona solução degermante ${ }^{5}$ embebido em gaze, seguido por álcool iodado $^{6}$. Os testículos foram firmemente estabilizados na região escrotal para incisão cutânea diretamente sobre cada testículo, no aspecto mais ventral do saco escrotal. Em seguida, incisou-se a túnica vaginal parietal, expondo o testículo cuidadosamente com discreta tração. Com auxílio de uma pinça hemostática, divulsionou-se o ligamento da cauda do epidídimo para liberação da túnica vaginal visceral e exposição dos vasos espermáticos, efetuando assim a técnica aberta proposta para ligadura e hemostasia. Até essa etapa, os procedimentos anestésicos e cirúrgicos foram os mesmos para todos os animais, diferenciando-se, a partir de então, de acordo com o tipo de oclusão do cordão espermático.

Os animais foram divididos aleatoriamente em três grupos de 13 animais. No grupo I (GI), os animais foram submetidos à orquiectomia utilizando-se ligadura do cordão espermático com fio cirúrgico. Após o cordão ter sido pinçado com duas pinças hemostáticas retas, seccionou-se entre as mesmas para remover o testículo. Em seguida, proximal à pinça remanescente, realizou-se ligadura e sobreligadura com fio categute cromado $\mathrm{n}^{\circ} 2-0^{7}$, englobando cordão vascular e ducto deferente. A pinça foi então retirada, observando-se a adequada hemostasia (Figura 1).

No grupo II (GII), utilizou-se o próprio cordão espermático para realização de um nó em figura de oito. Após passar o cordão espermático sobre os ramos de uma pinça hemostática reta, girou-se o instrumental por baixo do próprio cordão, pinçando sua porção mais distal e seccionou-a para remover o testículo. O nó em figura de oito foi finalizado ao retra- 
ir-se a pinça com a extremidade do cordão espermático através das laçadas realizadas (Figura 2).

Nos animais do grupo III (GIII), o ducto deferente e os vasos espermáticos foram utilizados para realização de nós quadrados. Após realizar a dissecação do ducto deferente e secção da sua inserção ao testículo, segurou-se esse segmento em uma mão e o testículo unido ao cordão vascular em outra, realizando assim três nós quadrados simples com essas estruturas. Em seguida, o cordão vascular aderido ao testículo foi seccionado distalmente aos nós (Figura 3).

Independente da técnica empregada, a ferida cirúrgica na bolsa escrotal não foi suturada, permitindo a cicatrização por segunda intenção. No perío-
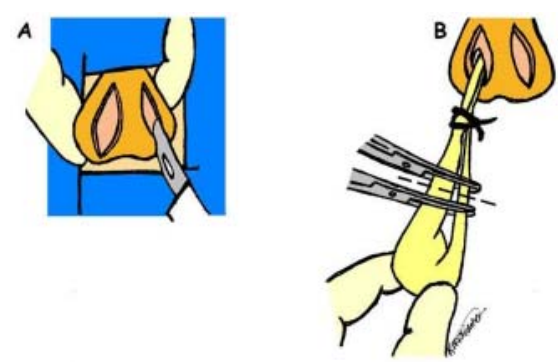

Figura 1. Técnica aberta de orquiectomia utilizando fio cirúrgico para ligadura do cordão espermático. (A) Duas incisões paralelas e longitudinais são realizadas na bolsa escrotal sobre os testículos. (B) Após abertura das túnicas vaginais seguida pela exposição testicular, são colocadas duas pinças hemostáticas retas sobre o cordão espermático. Incisa-se entre as pinças para remoção testicular (linha tracejada), seguido pela realização da ligadura e sobreligadura proximalmente à pinça restante com fio cirúrgico absorvível $n^{\circ} 2-0$.
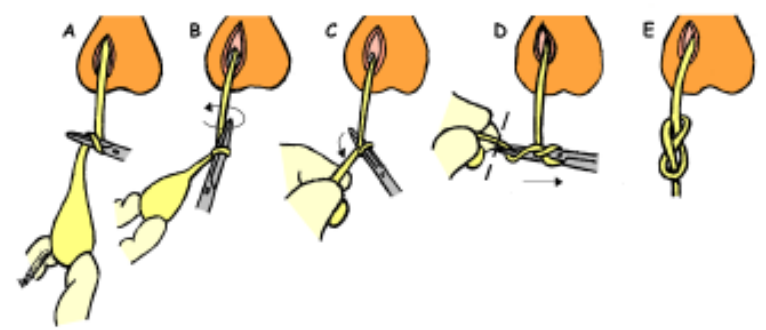

Figura 2. Técnica aberta de orquiectomia utilizando nó em figura de oito. (A) O cordão espermático é passado sobre os ramos de uma pinça hemostática reta. (B) Após o instrumental ter sido girado por baixo do cordão, (C) sua porção mais distal, próxima ao testículo, é apreendida pela pinça. (D) O cordão é então seccionado (linha tracejada), promovendo a remoção testicular e o instrumental é, então, retraído através da laçadas (seta), (E) finalizando o nó em figura de oito. do pós-operatório, os gatos foram mantidos em gaiolas individuais, com colar elisabetano por tempo necessário à cicatrização cutânea. Foi administrado cetoprofeno $^{8}(1 \mathrm{mg} / \mathrm{kg}, \mathrm{IM}, \mathrm{SID})$ nos três primeiros dias após a cirurgia e realizada a limpeza da ferida cirúrgica com solução fisiológica ${ }^{9}$ e iodopovidona ${ }^{10}$ diariamente até completa cicatrização. Devido ao emprego de técnica asséptica, não foi realizada antibioticoterapia no período pós-operatório.

Os animais foram submetidos diariamente à avaliação clínica geral e observação de características da ferida cirúrgica, tais como presença de secreção, edema, seroma, hemorragia, hematoma ou infecção, até sua completa cicatrização. Nas variáveis paramétricas, utilizou-se o teste $\mathrm{F}$ para análise comparativa entre os grupos $(\mathrm{p} \leq 0,05)$ e as variáveis não paramétricas foram avaliadas pelo teste do qui-quadrado $\left(\chi^{2}\right)$ para cada dia separadamente $(\mathrm{p} \leq 0,05)$.

\section{RESULTADOS}

Nenhum dos animais apresentou hemorragia dos vasos espermáticos imediatamente após hemostasia. No período transoperatório, não se notou diferença significativa no tempo cirúrgico para as três diferentes técnicas de orquiectomia empregadas.

No período pós-operatório, a avaliação clínica geral mostrou-se pouco alterada e não foram observadas diferenças comportamentais em nenhum dos animais. Notou-se uma diferença estatística significativa entre os grupos quanto à frequência respiratória, observando-se uma elevação na média do GI durante o pós-operatório, apesar de esses valores esta-
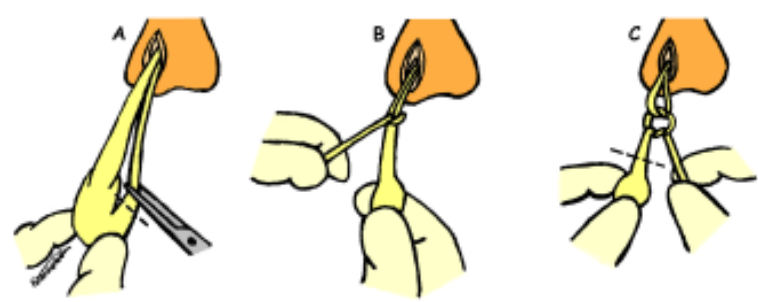

Figura 3. Técnica aberta de orquiectomia utilizando nós quadrados com o ducto deferente e os vasos espermáticos. (A) O ducto deferente é dissecado e seccionado próximo ao testículo (linha tracejada). (B) Com o ducto deferente em uma das mãos e o cordão vascular em outra, são realizados três nós quadrados simples. (C) Posteriormente, o cordão vascular é seccionado distalmente aos nós (linha tracejada), promovendo a remoção testicular. 
rem próximos aos limites fisiológicos para a espécie (Figura 4).

Em relação à temperatura corporal, não houve variação significativa entre os grupos, foi observada uma diminuição no primeiro dia após a intervenção cirúrgica em alguns animais, com retorno aos valores normais após o terceiro dia.

As variáveis não paramétricas analisadas não apresentaram diferença significativa entre os grupos. Foi observado clinicamente edema na bolsa escrotal de todos os animais. A ferida cirúrgica escrotal apresentou discreta secreção serosa no GI apenas no primeiro dia após a cirurgia e discreta secreção serossanguinolenta nos animais dos GII e GIII, nos dois primeiros dias de pós-operatório. Notou-se um aumento de volume com consistência firme e nodu-lar na base da bolsa escrotal nos animais do GI e uma consistência firme nas bordas da ferida cirúrgica em todos os grupos após a cicatrização completa. Características clínicas de infecção, seroma, hemorragia, hematoma ou quaisquer outras complicações pósoperatórias locais ou sistêmicas não foram observadas.

\section{DISCUSSÃO}

As três técnicas cirúrgicas mais utilizadas para orquiectomia em gatos [9] foram avaliadas nessa pesquisa. Embora a técnica aberta ofereça maior risco de contaminação, mostra-se vantajosa pelo fato da ligadura ser colocada diretamente ao redor do pedículo vascular $[10,14]$. Independente

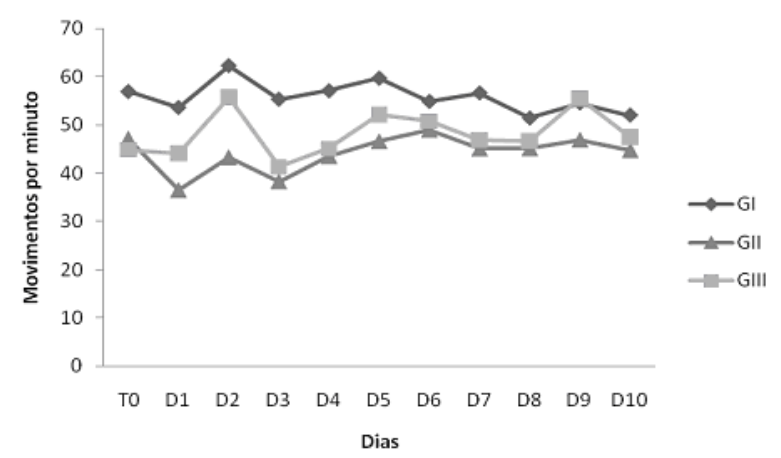

Figura 4. Médias da frequência respiratória dos gatos submetidos a diferentes técnicas de orquiectomia. (GI: técnica com fio cirúrgico; GII: técnica do nó em figura de oito; GIII: técnica dos nós quadrados; $\mathrm{T}_{0}$ : pré-operatório; D1 a D10: referente aos dias de pós-operatório). do método escolhido, deve-se assegurar uma técnica asséptica criteriosa, minimizando o risco de infecção [4].

Em todos os animais estudados, a exposição testicular foi realizada por meio de incisão na pele escrotal, separadamente sobre cada testículo, já que na técnica de orquiectomia em gatos não se deve realizar uma incisão única no septo escrotal, pois pode ocasionar hemorragia acentuada e favorecer a formação de hematomas [6]. Além disso, optou-se nesse estudo por não suturar a ferida cirúrgica, para proporcionar drenagem adequada das secreções e prevenir a formação de seromas $[1,9]$.

Apesar da variável frequência respiratória diferir estatisticamente entre os grupos, apresentando-se mais elevada no GI, foi um dado pouco expressivo clinicamente, pois os outros parâmetros avaliados permaneceram dentro dos valores fisiológicos para a espécie felina. Esses dados clínicos refletem a inexistência de complicações importantes decorrentes da cirurgia, tais como infecção, dor, hipotensão, hipovolemia ou choque [12]. Em relação à temperatura corporal, a diminuição observada em alguns animais no primeiro dia após a intervenção cirúrgica é de ocorrência comum, pois a hipotermia no pós-operatório imediato ocorre devido à ação dos anestésicos ao deprimir o metabolismo [18,19, 26].

A literatura relata que o cordão espermático pode ser ligado com fio de sutura ou, preferivelmente, pode ser ligado com ele próprio, evitando a colocação de materiais exógenos em um ambiente potencialmente contaminado, pelo contato com urina e fezes [5]. A técnica de ligadura com fio cirúrgico é indicada para orquiectomia em gatos muito jovens, pois permite a hemostasia satisfatória dos vasos espermáticos $[1,15]$. Os mesmos autores contraindicam as técnicas do nó em figura de oito e nós quadrados devido aos vasos serem extremamente pequenos e frágeis em filhotes. Na atual pesquisa, foram utilizados somente gatos adultos e as estruturas do cordão espermático se mostraram resistentes e eficientes para ligadura dos vasos testiculares com as técnicas empregadas.

Embora estatisticamente as variáveis qualitativas não tenham apresentado diferença significativa entre os grupos, observou-se clinicamente que houve maior edemaciação no grupo da técnica da liga- 
dura com fio cirúrgico. $\mathrm{O}$ fio categute, atuando como corpo estranho, interfere na reação inflamatória, tempo de absorção e cicatrização da ferida [11,21]. A estrutura de consistência firme observada na base da bolsa escrotal nos animais do GI provavelmente foi devido à reação inflamatória mais exacerbada causada pela presença do fio [7]. Entretanto, não foram observadas complicações como hemorragias, infecções e formação de abscessos relatadas nesse tipo de técnica $[2,16]$, demonstrando que o fio de sutura não foi um fator predisponente a complicações dessa natureza. Essa técnica não apresentou vantagens em relação às demais, além de existir o custo do fio de sutura e a possibilidade de contaminação por se usar um corpo estranho exógeno [14]. Além disso, pode ser uma técnica mais demorada [21], já que o fio categute pode romper-se ao cerrar o nó, por sua resistência tênsil ser outra propriedade de alta variabilidade.

Observou-se que a técnica do nó em figura de oito é um procedimento seguro e eficaz, conforme citado por Hay [8] e Howe [14]. Ao contrário do que Aronhson e Fagella [1] afirmaram da possibilidade de hemorragia grave ao utilizar essa técnica devido à frouxidão do nó, isso não foi verificado em nenhum dos animais do estudo. Apesar de ser eficaz, observou-se que a técnica em figura de oito exige maior habilidade do cirurgião quando comparada às demais técnicas avaliadas.

De acordo com Mandelker [17], a técnica dos nós quadrados utilizando o ducto deferente para ligadura dos vasos espermáticos mostrou-se simples e eficaz, com mínimo trauma tecidual e não apresentou complicações relacionadas à infecção ou hemorragia. Por outro lado, Blake [2] relata que a desvantagem desse procedimento seria exatamente o excesso de manuseio dos tecidos, podendo levar à hemorragia e edemaciação dos mesmos. O presente estudo demonstrou que a manipulação cautelosa dos tecidos e do processo de dissecação do ducto deferente ocasionou pouca manipulação tecidual sem lesionar os vasos espermáticos, demonstrada pela ausência de hemorragia e mínima reação inflamatória. Essa técnica mostrou-se eficaz, sem alterações pós-operatórias e de fácil execução.

As complicações graves após a orquiectomia em gatos são pouco frequentes mas eventualmente podem ocorrer funiculites, infecções, abscessos e aderências [3,15,20,24]. Entretanto, um estudo apontou que a frequência de complicações pós-operatórias clinicamente relevantes de cirurgias eletivas em gatos é importante e pode chegar a 12,2\% [20]. Isso pode ser justificado pela falta de acompanhamento pós-cirúrgico dos animais submetidos a esse tipo de procedimento, cujos pacientes muitas vezes recebem alta imediatamente após a recuperação anestésica, dificultando a identificação e subestimando as complicações pós-operatórias. Essas alterações não foram observadas em nenhum dos animais avaliados que foram acompanhados por no mínimo 10 dias, demonstrando a eficácia das técnicas cirúrgicas utilizadas.

No presente estudo, os resultados da comparação das três técnicas abertas empregadas para hemostasia dos vasos espermáticos na orquiectomia de gatos não demonstraram diferença significativa. Entretanto, a técnica dos nós quadrados foi a que se mostrou mais eficaz, sem quaisquer alterações pósoperatórias e a de mais fácil execução quando comparada às demais. Sugere-se que o principal fator responsável pelo sucesso dos procedimentos seja a manutenção dos princípios fundamentais de uma técnica cirúrgica pouco traumática e asséptica, com rigorosa antissepsia, absoluto controle do sangramento, precisa dissecação anatômica e manipulação cuidadosa dos tecidos. Além disso, o acompanhamento do paciente no período pós-operatório pode prevenir incidentes ocasionais tardios decorrentes da intervenção cirúrgica, corrigindo-os precocemente.

\section{CONCLUSÃO}

As três técnicas cirúrgicas avaliadas se mostraram exequíveis e eficientes para realização de orquiectomia em gatos adultos. Entretanto, a técnica dos nós quadrados utilizando-se o ducto deferente e os vasos espermáticos foi a que se mostrou mais eficaz, sem quaisquer alterações pós-operatórias importantes e a de mais fácil execução quando comparada às demais.

\section{NOTAS INFORMATIVAS}

${ }^{1}$ Benzilpenicilina $600.000^{\circledR}$, Laboratório Teuto Brasileiro S.A., Anápolis, GO.

${ }^{2}$ Acepran $1 \%{ }^{\circledR}$, Univet S.A. Indústria Veterinária, São Paulo, SP.

${ }^{3}$ Ketamin $5 \%{ }^{\circledR}$, Cristália Produtos químicos farmacêuticos Ltda., Itapira, SP. 
${ }^{4}$ Rompun $2 \%{ }^{\circledR}$, Bayer S.A. Saúde Animal, São Paulo, SP.

${ }^{5}$ Biotrat Iodopovidona degermante ${ }^{\circledR}$, LM Farma Indústria e Comércio Ltda., São José dos Campos, SP.

${ }^{6}$ Rialcool ${ }^{\circledR,}$ Indústria farmacêutica Rioquímica Ltda., São José do Rio Preto, SP.

${ }^{7}$ Fio Cirúrgico Brasuture ${ }^{\circledR}$, Brasuture Indústria e Comércio
Ltda., São Sebastião da Gama, SP.

${ }^{8}$ Ketoflex $1 \%{ }^{\circledR}$, Mundo Animal Laboratório Veterinário Ltda., São Paulo, SP.

${ }^{9}$ Solução de Cloreto de Sódio 0,9\% ${ }^{\circledR}$, Laboratório Sanobiol Ltda., Pouso Alegre, MG.

${ }^{10}$ Biotrat Iodopovidona tópica ${ }^{\circledR}$, LM Farma Indústria e Comércio Ltda., São José dos Campos, SP.

\section{REFERÊNCIAS}

1 Aronsohn M.G. \& Faggella A.M. 1993. Surgical techniques for neutering 6 to 14 week-old kittens. Journal of the American Veterinary Medical Association. 202(1): 53-55.

2 Blake J.A. 1969. A technique for castrating cats. Journal of the American Veterinary Medical Association. 154(1): 25-26.

3 Bloomberg M.S. 1996. Surgical neutering and nonsurgical alternatives. Journal of the American Veterinary Medical Association. 208(4): 517-519.

4 Boothe H.W. 2003. Testes and epididymides. In: Slatter D. (Ed). Textbook of Small Animal Surgery. 3rd edn. Philadelphia: Saunders, pp. 1521-1530.

5 Cockshutt J. 2003. Principles of surgical asepsis. In: Slatter D. (Ed). Textbook of Small Animal Surgery. 3rd edn. Philadelphia: Saunders, pp. 149-155.

6 Crane S.W. 1996. Orquiectomia de testículos descidos e retidos no cão e no gato. In: Bojrab M.J. (Ed). Técnicas atuais em cirurgia de pequenos animais. 3.ed. São Paulo: Roca, pp.391-397.

7 Fossum T.W. 2005. Biomateriais, sutura e hemostasia. In: Fossum T.W., Hedlund C.S., Hulse D.A., Johnson A.L., Seim H.B., Willard M.D. \& Carroll G.L. (Eds). Cirurgia de pequenos animais. 2.ed. São Paulo: Roca, pp. 45-60.

8 Hay C.W. 1996. Cat castration: an easy way to knot the spermatic cord. Veterinary Medicine. 91: 702-703.

9 Hedlund C.S. 2005. Cirurgia do sistema tegumentar. In: Fossum T.W., Hedlund C.S., Hulse D.A., Johnson A.L., Seim H.B., Willard M.D. \& Carroll G.L (Eds). Cirurgia de pequenos animais. 2.ed. São Paulo: Roca, pp. 135-230.

10 Hedlund C.S. 2007. Surgery of the reproductive and genital systems. In: Fossum T.W., Hedlund C.S., Hulse D.A., Johnson A.L., Seim H.B., Willard M.D. \& Carroll G.L (Eds). Small animal surgery. 3rd edn. Missouri: Mosby, pp. 702-774.

11 Hering F.L.O., Gabor S. \& Rosenberger D. 1993. Bases técnicas e teóricas de fios e suturas. São Paulo: Roca, $232 \mathrm{p}$.

12 Houston D.M. \& Radostits O.M. 2002. O exame clínico. In: Radostits O.M., Mayhew I.G.J. \& Houston D.M. (Eds). O exame clínico e diagnóstico em veterinária. Rio de Janeiro: Guanabara Koogan, pp. 71-97.

13 Howe L.M. 1997. Short-term results and complications of prepuberal gonadectomy in cats and dogs. Journal of the American Veterinary Medical Association. 211(1): 57-62.

14 Howe, L.M. 2006. Surgical methods of contraception and sterilization. Theriogenology. 66(3): 500-509.

15 Kustritz M.V.R. 1999. Early spay-neuter in the dog and cat. Veterinary Clinic North America: Small Animal Practice. 29(4): 935-943.

16 Kustritz M.V.R. 2002. Early spay-neuter: clinical considerations. Clinical Techniques in Small Animal Practice. 17(3): 124-128.

17 Mandelker L. 1978. A sterile surgical procedure for feline castration (a photography essay). Veterinary Medicine: Small Animal Clinician. 73(7): 904-905.

18 Miller J.B. 2004. Hipertermia e hipotermia. In: Ettinger J.S. \& Feldman E.C. (Eds). Tratado de medicina interna veterinária. 5.ed. Rio de Janeiro: Guanabara Koogan, pp. 6-10.

19 Pascoe P.J. 1998. Cuidados pós-operatórios do paciente. In: Slatter D. (Ed). Manual de cirurgia de pequenos animais. 2.ed. São Paulo: Manole, pp. 287-299.

20 Pollari F.L. \& Bonnett B.N. 1996. Evaluation of postoperative complications following elective surgeries of dogs and cats at private practices using computer records. Canadian Veterinary Journal. 37(11): 672-678.

21 Silva L.A.F., França R.O., Vieira D., Sousa V.R., Franco L.G., Moura M.I., Silva M.A.M., Trindade B.R., Costa G.L. \& Bernardes K.M. 2006. Emprego da abraçadeira de náilon na orquiectomia em equinos. Acta Scientiae Veterinariae. 34(3): 261-266.

22 Smeak D.D. 2003. Abdominal hernias. In: Slatter D. (Ed). Textbook of Small Animal Surgery. 3rd edn. Philadelphia: Saunders, pp. 449-470. 
23 Stubbs W.P. \& Bloomberg M.S. 1995. Implications of early neutering in the dog and cat. Seminars in Veterinary Medicine and Surgery (Small Animal). 10(1): 8-12.

24 Swalec K.M. \& Smeak D.D. 1989. Priapism after castration in a cat. Journal of the American Veterinary Medical Association. 195(7): 963-964.

25 Tivers M.S., Travis T.R., Windsor R.V. \& Hotston A. M. 2005. Questionnaire study of canine neutering techniques taught in UK veterinary schools and those used in practice. Journal of Small Animal Practice. 46(9): 423-424.

26 Yazbek K.V.B. 2002. Hipotermia. In: Fantoni T.F. \& Cortopassi S.R.G. (Eds). Anestesia em cães e gatos. São Paulo: Roca, pp. 373-378. 
